\title{
Digital Lusternik-Schnirelmann category of digital functions
}

\author{
Tane Vergili ${ }^{1}\left(\right.$ ), Ayşe Borat ${ }^{* 2}$ (1) \\ ${ }^{1}$ Karadeniz Technical University, Faculty of Science, Department of Mathematics, Trabzon, Turkey \\ ${ }^{2}$ Bursa Technical University, Faculty of Engineering and Natural Sciences, Department of Mathematics, \\ Bursa, Turkey
}

\begin{abstract}
Roughly speaking, the digital Lusternik-Schnirelmann category of digital images studies how far a digital image is away from being digitally contractible. The digital LusternikSchnirelmann category (digital LS category, for short) is defined in [A. Borat and T. Vergili, Digital Lusternik-Schnirelmann category, Turkish J. Math. 2018]. In this paper, we introduce the digital LS category of digital functions. We will give some basic properties and discuss how this new concept will behave if we change the adjacency relation in the domain and in the image of the digital function and discuss its relation with the digital LS category of a digital image.
\end{abstract}

Mathematics Subject Classification (2010). 55M30, 68U10

Keywords. Lusternik Schnirelmann category, digital topology

\section{Introduction}

The Lusternik-Schnirelmann category of a space $X$, first introduced in [21], is the least number $n$ such that there is an open cover $\left\{U_{1}, \ldots, U_{n+1}\right\}$ of $X$ with the property that the inclusion $i_{j}: U_{j} \hookrightarrow X$ is nullhomotopic in $X$ for each $j$. For more details, see [11]. The Lusternik-Schnirelmann category of maps is first introduced in [14] and is studied in detail in [1]. For more details see $[11,18,24]$ and for a simplicial analog see [23].

The digital LS category of a digital image is introduced in [3] and its close relative, digital topological complexity, is introduced in [19]. For the definition of usual topological complexity and its basic properties, see [13].

This paper is organized as follows. In Section 2, we will recall some basic definitions and theorems from digital topology that we will use throughout this paper. We will also recall the definition of the digital LS category of a digital image and discuss digital LS category of simple closed $\kappa$-curves.

In Section 3, we will introduce the definition of the digital LS category of a digital function and introduce the main theorems of this paper, answering questions such as

\footnotetext{
*Corresponding Author.

Email addresses: tane.vergili@ktu.edu.tr (T. Vergili), ayse.borat@btu.edu.tr (A. Borat)

Received: 02.05.2019; Accepted: 27.10.2019
} 
how digitally homotopic functions and digital homotopy equivalence affect the digital Lusternik-Schnirelmann category of the given functions or the digital images. Moreover, relations between the digital LS category of a digital function and the digital LS category of a digital image are studied in this section and Section 4.

\section{Background}

In this section we will recall some basic definitions and theorems from digital topology and recall the digital Lusternik-Schnirelmann category of digital images. At the end of this section we will give a discussion on simple closed $\kappa$-curves and compute their digital LS category.

A digital image $X$ is a subset of $\mathbb{Z}^{n}$ for a positive integer $n$. We impose an adjacency relation on $\mathbb{Z}^{n}$ in order to work on a digital image $X \subset \mathbb{Z}^{n}$ as follows [7]: Let $p=$ $\left(p_{1}, p_{2}, \ldots, p_{n}\right)$ and $q=\left(q_{1}, q_{2}, \ldots, q_{n}\right)$ be two points in $\mathbb{Z}^{n}$. Then for $1 \leq \ell \leq n, p$ and $q$ are said to be $c_{\ell}$-adjacent whenever

- there are at most $\ell$ indices $i$ such that $\left|p_{i}-q_{i}\right|=1$ and

- $p_{j}=q_{j}$ for all other indices $j$ satisfying $\left|p_{i}-q_{i}\right| \neq 1$.

Note that $c_{\ell}$ indicates the number of adjacent points in $\mathbb{Z}^{n}$, according to this adjacency. For instance, we have $c_{1}=2$ in $\mathbb{Z}, c_{1}=4$ and $c_{2}=8$ in $\mathbb{Z}^{2}$, and $c_{1}=6, c_{2}=18, c_{3}=26$ in $\mathbb{Z}^{3}$. We usually denote an adjacency relation by Greek letters such as $\kappa, \lambda$, etc., and a digital image by a pair $(X, \kappa)$ where $X \subseteq \mathbb{Z}^{n}$ and $\kappa$ is an adjacency relation inherited from $\mathbb{Z}^{n}$.

A digital image $(X, \kappa)$ is said to be $\kappa$-connected if for any pair of elements $x$ and $x^{\prime}$ in $X$, there exists a sequence $\left\{x_{i}\right\}_{i=0}^{n} \subset X$ such that $x=x_{0}, x^{\prime}=x_{n}$ and $x_{i}$ and $x_{i+1}$ are $\kappa$-adjacent for $0 \leq i<n[17]$.

In [4], a digital interval is defined as a subset of $\mathbb{Z}$ of the form

$$
[a, b]_{\mathbb{Z}}=\{n \in \mathbb{Z} \mid a \leq n \leq b\} .
$$

where 2-adjacency is assumed.

Definition 2.1. ([5]) Let $(X, \kappa)$ and $(Y, \lambda)$ be digital images. A function $f: X \rightarrow Y$ is $(\kappa, \lambda)$-continuous if $f(x)$ and $f\left(x^{\prime}\right)$ are $\lambda$-adjacent or equal in $Y$ whenever $x$ and $x^{\prime}$ are $\kappa$-adjacent in $X$.

Let $(X, \kappa)$ and $(Y, \lambda)$ be digital images. A function $f: X \rightarrow Y$ is called $(\kappa, \lambda)$ isomorphism [8] (called a homeomorphism rather than isomorphism in [4,5]) if $f$ is $(\kappa, \lambda)$ continuous and bijective and further its inverse $f^{-1}: Y \rightarrow X$ is $(\lambda, \kappa)$-continuous.

Theorem 2.2. ([5]) Let $f: X \rightarrow Y$ and $g: Y \rightarrow Z$ be $\left(\kappa_{0}, \kappa_{1}\right)$-continuous and $\left(\kappa_{1}, \kappa_{2}\right)$ continuous functions respectively. Then the composite function $g \circ f: X \rightarrow Z$ is $\left(\kappa_{0}, \kappa_{2}\right)$ continuous.

Definition 2.3. ([5,20]) Let $f, g: X \rightarrow Y$ be $(\kappa, \lambda)$-continuous functions. If there exist a positive integer $m$ and a function

$$
F: X \times[0, m]_{\mathbb{Z}} \rightarrow Y
$$

with the following conditions, then $F$ is said to be a $(\kappa, \lambda)$-homotopy, and $f$ and $g$ are called $(\kappa, \lambda)$-homotopic in $Y$ (denoted by $f \simeq_{\kappa, \lambda} g$ ).

(i) For all $x \in X, F(x, 0)=f(x)$ and $F(x, m)=g(x)$.

(ii) For all $x \in X$, the induced function $F_{x}:[0, m]_{\mathbb{Z}} \rightarrow Y$ defined by $F_{x}(t)=F(x, t)$ is $(2, \lambda)$-continuous.

(iii) For all $t \in[0, m]_{\mathbb{Z}}$, the induced function $F_{t}: X \rightarrow Y$ defined by $F_{t}(x)=F(x, t)$ is $(\kappa, \lambda)$-continuous. 
Definition 2.4. ([6]) A $(\kappa, \lambda)$-continuous map $f: X \rightarrow Y$ is said to be a $(\kappa, \lambda)$-homotopy equivalence if there exists a $(\lambda, \kappa)$-continuous map $g: Y \rightarrow X$ such that $g \circ f \simeq_{\kappa, \kappa} \operatorname{Id}_{X}$ and $f \circ g \simeq_{\lambda, \lambda} \operatorname{Id}_{Y}$ where $\operatorname{Id}_{X}$ and $\operatorname{Id}_{Y}$ are the identity maps on $X$ and $Y$ respectively. We say $X$ and $Y$ are $(\kappa, \lambda)$-homotopy equivalent if there is a $(\kappa, \lambda)$-homotopy equivalence from $X$ to $Y$.

We call a $(\kappa, \lambda)$-continuous map $f: X \rightarrow Y(\kappa, \lambda)$-nullhomotopic if it is $(\kappa, \lambda)$-homotopic to a constant function $c: X \rightarrow Y, c(x)=c_{0}$ for some $c_{0} \in Y$ [4].

Throughout the paper, a cover of a digital image $(X, \kappa)$ means a collection of subsets $\left\{U_{i}\right\}$ of $X$ whose union equals $X$.

Definition 2.5. ([3]) The digital LS category of a digital image $(X, \kappa)$ is the least integer $n$ such that there exists a cover $\left\{U_{1}, U_{2}, \ldots, U_{n+1}\right\}$ of $X$ where each inclusion map $i_{i}$ : $U_{i} \hookrightarrow X$ for $i=1, \ldots, n+1$ is $(\kappa, \kappa)$-nullhomotopic (or $\kappa$-nullhomotopic for short) in $X$. This will be denoted by $\operatorname{cat}_{\kappa}(X)=n$.

Notice that $\operatorname{cat}_{\kappa}(X)$ can be at most the number of lattice points in $X$.

The following definition is given in [10]. Suppose $\kappa_{1}$ and $\kappa_{2}$ are two adjacency relations on a set $X$. Then we say that $\kappa_{1}$ dominates $\kappa_{2}, \kappa_{1} \geq_{d} \kappa_{2}$, if for $x, x^{\prime} \in X$, if $x$ and $x^{\prime}$ are $\kappa_{1}$-adjacent then $x$ and $x^{\prime}$ are $\kappa_{2}$-adjacent.

Remark 2.6. Suppose $u \leq v$. In [3], the authors used the notations " $\kappa \leq \lambda^{\prime \prime}$ or " $\lambda \geq \kappa^{\text {" }}$ in such a way that " $c_{u} \leq c_{v}$ "; however, by the definition of these notations in [10] we have " $c_{v} \leq c_{u}$ ". For consistency in the literature, we have chosen to use the definition of [10].

As in the traditional algebraic topology setting, the digital LS category is a homotopy invariant in the digital sense.

Theorem 2.7. ([3]) If the digital images $(X, \kappa)$ and $(Y, \lambda)$ are $(\kappa, \lambda)$-homotopy equivalent, then $\operatorname{cat}_{\kappa}(X)=\operatorname{cat}_{\lambda}(Y)$.

A simple closed $\kappa$-curve $S$ in a digital image $(X, \kappa)$ is a sequence $\left\{x_{i}\right\}_{i=0}^{m-1}$ for $m \geq 4$ in $X$ such that $x_{i}$ and $x_{j}$ are $\kappa$-adjacent if and only if $j=(i \pm 1) \bmod m$ [5].

Recall that if the identity map $\operatorname{Id}_{X}: X \rightarrow X$ is $(\kappa, \kappa)$-homotopic to a constant function $c: X \rightarrow X, c(x)=c_{0}$ for some $c_{0}$ in $X$, then $(X, \kappa)$ is called $\kappa$-contractible $[4,20]$.

Theorem 2.8. ([9]) A simple closed $\kappa$-curve $S$ which contains more than four points is not $\kappa$-contractible.

However, if we remove a point from a simple closed $\kappa$-curve $S$ with $|S|>4$, we get a $\kappa$-contractible digital image.

Proposition 2.9. Let $S=\left\{x_{i}\right\}_{i=0}^{m}$ be a simple closed $\kappa$-curve with $m \geq 4$. Then $S \backslash\left\{x_{m}\right\}$ is $\kappa$-contractible.

Proof. The desired digital $\kappa$-homotopy function between the identity map and a constant map on $S \backslash\left\{x_{m}\right\}$ as follows.

$$
\begin{aligned}
H: S \backslash\left\{x_{m}\right\} \times[0, m-1]_{\mathbb{Z}} & \rightarrow S \backslash\left\{x_{m}\right\} \\
\left(x_{i}, t\right) & \mapsto H\left(x_{i}, t\right)= \begin{cases}x_{i-t} & 0 \leq t \leq i \\
x_{0} & \text { otherwise }\end{cases}
\end{aligned}
$$

Theorem 2.10. Let $S$ be a simple closed $\kappa$-curve with $|S|>4$. Then cat $_{\kappa}(S)=1$. 
Proof. Let $S=\left\{x_{i}\right\}_{i=0}^{m}$ with $m \geq 4$. Then $\operatorname{cat}_{\kappa}(S)>0$ since $S$ is not $\kappa$-contractible. Consider the subsets of $S, U_{1}=\left\{x_{m}\right\}$ and $U_{2}=S \backslash\left\{x_{m}\right\}$. Then the inclusion map $i_{i}: U_{i} \rightarrow S$ for $i=1,2$ is $\kappa$-nullhomotopic since $U_{1}$ is a singleton set and $U_{2}$ is $\kappa$ contractible by Proposition 2.9.

\section{Digital Lusternik-Schnirelmann category of digital functions}

Definition 3.1. The digital LS category of a $(\kappa, \lambda)$-continuous function $f: X \rightarrow Y$ is defined to be the least integer $n$ such that there is a cover $\left\{U_{1}, \ldots, U_{n+1}\right\}$ of $X$ such that $\left.f\right|_{U_{j}}$ is $(\kappa, \lambda)$-nullhomotopic for each $j$.

We will denote it by $\operatorname{cat}_{\kappa, \lambda}(f)$.

Notice that $\operatorname{cat}_{\kappa, \lambda}(f)$ can be no more than the number of lattice points of $X$.

Remark 3.2. It is obvious that $\operatorname{cat}_{\kappa}(X)=\operatorname{cat}_{\kappa, \kappa}\left(\operatorname{Id}_{X}\right)$ (it follows from the definition of digital LS category).

Proposition 3.3. Suppose $\kappa_{1}$ and $\kappa_{2}$ are two adjacency relations on a set $X$ with $\kappa_{1} \geq_{d} \kappa_{2}$ and $\lambda$ is an adjacency relation on a set $Y$. If $f$ is $\left(\kappa_{1}, \lambda\right)$-continuous then cat $_{\kappa_{1}, \lambda}(f) \geq$ $\operatorname{cat}_{\kappa_{2}, \lambda}(f)$.

Proof. Since $\kappa_{1} \geq_{d} \kappa_{2}$, a function that is $\left(\kappa_{1}, \lambda\right)$-continuous is $\left(\kappa_{2}, \lambda\right)$-continuous. Therefore, $f$ is $\left(\kappa_{2}, \lambda\right)$-continuous, and $\left(\kappa_{1}, \lambda\right)$-homotopies are $\left(\kappa_{2}, \lambda\right)$-homotopies. The assertion follows easily from Definition 3.1.

Proposition 3.4. Suppose $\lambda_{1}$ and $\lambda_{2}$ are two adjacency relations on a set $Y$ with $\lambda_{2} \geq_{d} \lambda_{1}$ and $\kappa$ is an adjacency relation on a set $X$. If $f$ is $\left(\kappa, \lambda_{2}\right)$-continuous then cat $_{\kappa, \lambda_{1}}(f) \leq$ $\operatorname{cat}_{\kappa, \lambda_{2}}(f)$

Proof. Since $f$ is $\left(\kappa, \lambda_{2}\right)$-continuous and $\lambda_{2} \geq_{d} \lambda_{1}, f$ is also $\left(\kappa, \lambda_{1}\right)$-continuous. Let cat $_{\kappa, \lambda_{2}}(f)=n$. Then there are $U_{1}, \ldots, U_{n+1}$ subsets of $X$ covering $X$ such that $\left.f\right|_{U_{j}}$ : $U_{j} \rightarrow X$ is $\left(\kappa, \lambda_{2}\right)$-nullhomotopic, for each $j$. That is, there is a $\left(\kappa, \lambda_{2}\right)$-homotopy $H^{j}$ : $U_{j} \times[0, m]_{\mathbb{Z}} \rightarrow Y$ between $\left.f\right|_{U_{j}}$ and a constant function $c_{j}:\left(U_{j}, \kappa\right) \rightarrow\left(Y, \lambda_{2}\right)$. If we consider $\lambda_{1}$-adjacency on $Y$ and consider $G^{j}: U_{j} \times[0, m]_{\mathbb{Z}} \rightarrow Y$ defined by $G^{j}(x, t)=H^{j}(x, t)$, the function $G^{j}$ becomes a $\left(\kappa, \lambda_{1}\right)$-homotopy which follows from the idea that two $\lambda_{2}$-adjacent points are $\lambda_{1}$-adjacent since $\lambda_{2} \geq_{d} \lambda_{1}$. Thus cat ${ }_{\kappa, \lambda_{1}}(f) \leq n$.

Example 3.5. Let $(X, \kappa)$ and $(Y, \lambda)$ be digital images. If $f: X \rightarrow Y$ is $(\kappa, \lambda)$-nullhomotopic, $\operatorname{cat}_{\kappa, \lambda}(f)=0$.

Theorem 3.6. Suppose $X$ is $\kappa$-connected and $Y$ is $\lambda$-connected. If $f: X \rightarrow Y$ is a $(\kappa, \lambda)$-continuous function, then $\operatorname{cat}_{\kappa, \lambda}(f) \leq \min \left\{\operatorname{cat}_{\kappa}(X), \operatorname{cat}_{\lambda}(Y)\right\}$.

Proof. Let $\operatorname{cat}_{\kappa}(X)=n$. Then there is a cover $\left\{U_{1}, \ldots, U_{n+1}\right\}$ of $X$ such that the inclusion $i_{j}: U_{j} \hookrightarrow X$ is $\kappa$-nullhomotopic in $X$ for each $j$. So for each $j$, we can write a $(\kappa, \kappa)$-homotopy

$$
H^{j}: U_{j} \times[0, m]_{\mathbb{Z}} \rightarrow X \quad \text { satisfying }
$$

(1a) For all $x \in U_{j}, H^{j}(x, 0)=i_{j}(x)$ and $H^{j}(x, m)=c_{j}$ for some $c_{j} \in X$.

(1b) For all $x \in U_{j}, H_{x}^{j}:[0, m]_{\mathbb{Z}} \rightarrow X$ defined by $H_{x}^{j}(t)=H^{j}(x, t)$ is $(2, \kappa)$-continuous.

(1c) For all $t \in[0, m]_{\mathbb{Z}}, H_{t}^{j}: U_{j} \rightarrow X$ defined by $H_{t}^{j}(x)=H^{j}(x, t)$ is $(\kappa, \kappa)$-continuous.

Define functions as follows.

$$
\begin{aligned}
F^{j}: U_{j} \times[0, m]_{\mathbb{Z}} & \rightarrow Y \\
(x, t) & \rightarrow F^{j}(x, t)=f\left(H^{j}(x, t)\right)
\end{aligned}
$$


If we verify that the following three conditions hold (i.e., $F^{j}$ is a $(\kappa, \lambda)$-homotopy), then it follows that $\operatorname{cat}_{\kappa, \lambda}(f) \leq \operatorname{cat}_{\kappa}(X)$.

(2a) For all $x \in U_{j}, F^{j}(x, 0)=f\left(H^{j}(x, 0)\right)=f\left(i_{j}(x)\right)=\left.f\right|_{U_{j}}(x)$ and $F^{j}(x, m)=$ $f\left(H^{j}(x, 1)\right)=f\left(c_{j}\right)$ is constant in $Y$.

(2b) For all $x \in U_{j}, F_{x}^{j}:[0, m]_{\mathbb{Z}} \rightarrow Y$ defined by $F_{x}^{j}(t)=F^{j}(x, t)=f\left(H_{x}^{j}(t)\right)$ is $(2, \lambda)$ continuous by Theorem 2.2 since it is a composition of a $(\kappa, \lambda)$-continuous map $f$ and a $(2, \kappa)$-continuous map $H_{x}^{j}$.

(2c) For all $t \in[0, m]_{\mathbb{Z}}, F_{t}^{j}: U_{j} \rightarrow Y$ defined by $F_{t}^{j}(x)=F^{j}(x, t)=f\left(H_{t}^{j}(x)\right)$ is $(\kappa, \lambda)$ continuous by Theorem 2.2 since it is a composition of a $(\kappa, \lambda)$-continuous map $f$ and a $(\kappa, \kappa)$-continuous map $H_{t}^{j}$.

For the second half of the proof, suppose that $\operatorname{cat}_{\lambda}(Y)=k$. Then there is a cover $\left\{V_{1}, \ldots, V_{k+1}\right\}$ of $Y$ such that the inclusion $i_{j}: V_{j} \hookrightarrow Y$ is $\lambda$-nullhomotopic in $Y$ for each $j$. So for each $j$, we can write a $(\lambda, \lambda)$-homotopy

$$
H^{j}: V_{j} \times[0, m]_{\mathbb{Z}} \rightarrow Y \quad \text { satisfying }
$$

(3a) For all $y \in V_{j}, H^{j}(y, 0)=i_{j}(y)$ and $H^{j}(y, m)=\bar{d}_{j}(y)=d_{j}$ where $\bar{d}_{j}: V_{j} \rightarrow Y$ defined by $\bar{d}_{j}(y)=d_{j}$ is a constant function for some $d_{j} \in Y$.

(3b) For all $y \in V_{j}, H_{y}^{j}:[0, m]_{\mathbb{Z}} \rightarrow Y$ defined by $H_{y}^{j}(t)=H^{j}(y, t)$ is $(2, \lambda)$-continuous.

(3c) For all $t \in[0, m]_{\mathbb{Z}}, H_{t}^{j}: V_{j} \rightarrow Y$ defined by $H_{t}^{j}(y)=H^{j}(y, t)$ is $(\lambda, \lambda)$-continuous.

Define functions as follows.

$$
\begin{aligned}
F^{j}: f^{-1}\left(V_{j}\right) \times & {[0, m]_{\mathbb{Z}} \rightarrow Y } \\
& (x, t) \rightarrow F^{j}(x, t)=H^{j}(f(x), t)
\end{aligned}
$$

If we verify that it is a $(\kappa, \lambda)$-homotopy, then it follows that cat ${ }_{\kappa, \lambda}(f) \leq \operatorname{cat}_{\lambda}(Y)$ and this completes the proof.

(4a) For all $x \in f^{-1}\left(V_{j}\right), F^{j}(x, 0)=H^{j}(f(x), 0)=i_{j}(f(x))=\left.f\right|_{f^{-1}\left(V_{j}\right)}(x)$ and $F^{j}(x, m)=$ $H^{j}(f(x), m)=\bar{d}_{j}(f(x))=d_{j}$ is constant in $Y$.

(4b) For all $x \in f^{-1}\left(V_{j}\right), F_{x}^{j}:[0, m]_{\mathbb{Z}} \rightarrow Y$ defined by $F_{x}^{j}(t)=F^{j}(x, t)=H_{f(x)}^{j}(t)$ is $(2, \lambda)$-continuous from $(3 \mathrm{~b})$.

(4c) For all $t \in[0, m]_{\mathbb{Z}}, F_{t}^{j}: f^{-1}\left(V_{j}\right) \rightarrow Y$ defined by $F_{t}^{j}(x)=F^{j}(x, t)=H_{t}^{j}(f(x))$ is $(\kappa, \lambda)$-continuous by Theorem 2.2 since it is a composition of a $(\kappa, \lambda)$ continuous map $f$ and a $(\lambda, \lambda)$-continuous map $H_{t}^{j}$.

Proposition 3.7. If $f: X \rightarrow Y$ is $(\kappa, \lambda)$-continuous and $g: Y \rightarrow Z$ is $(\lambda, \eta)$-continuous then $\operatorname{cat}_{\kappa, \eta}(g \circ f) \leq \min \left\{\operatorname{cat}_{\kappa, \lambda}(f), \operatorname{cat}_{\lambda, \eta}(g)\right\}$.

Proof. In the first half of the proof we show that cat $\kappa, \eta(g \circ f) \leq \operatorname{cat}_{\kappa, \lambda}(f)$. Let cat $\operatorname{cat}_{\kappa, \lambda}(f)=$ $n$. Then there is a cover $\left\{U_{1}, \ldots, U_{n+1}\right\}$ of $X$ such that $\left.f\right|_{U_{j}}: U_{j} \rightarrow Y$ is $(\kappa, \lambda)$ nullhomotopic (i.e., it is $(\kappa, \lambda)$-homotopic to a constant function $\left.c_{j}:\left(U_{j}, \kappa\right) \rightarrow(Y, \lambda)\right)$ for each $j$.

$\left.(g \circ f)\right|_{U_{j}}=g \circ\left(\left.f\right|_{U_{j}}\right) \simeq_{\kappa, \eta} g \circ c_{j} \simeq_{\kappa, \eta} \bar{c}_{j}$ where $\bar{c}_{j}:\left(U_{j}, \kappa\right) \rightarrow(Z, \eta)$ is a constant function. So $\operatorname{cat}_{\kappa, \lambda}(g \circ f) \leq n$.

In the second half of the proof, we show that $\operatorname{cat}_{\kappa, \eta}(g \circ f) \leq \operatorname{cat}_{\lambda, \eta}(g)$. Let cat $\cot _{\lambda, \eta}(g)=m$. Then there is a cover $\left\{V_{1}, \ldots, V_{m+1}\right\}$ of $Y$ such that $\left.g\right|_{V_{j}}: V_{j} \rightarrow Y$ is $(\lambda, \eta)$-nullhomotopic (i.e., it is $(\lambda, \eta)$-homotopic to a constant function $\left.d_{j}:\left(V_{j}, \lambda\right) \rightarrow(Z, \eta)\right)$ for each $j$. 
Take $U_{j}:=f^{-1}\left(V_{j}\right)$ for each $\mathrm{j}$. Notice that the union of $U_{j}$ 's is $X$.

For all $x \in f^{-1}\left(V_{j}\right)$, we have

$$
(g \circ f)(x)=g\left(\left.f\right|_{f^{-1}\left(V_{j}\right)}(x)\right)=\left.g\right|_{V_{j}}\left(\left.f\right|_{f^{-1}\left(V_{j}\right)}(x)\right)=\left(\left.\left.g\right|_{V_{j}} \circ f\right|_{f^{-1}\left(V_{j}\right)}\right)(x) .
$$

In other words, $\left.(g \circ f)\right|_{U_{j}}=\left.\left.g\right|_{V_{j}} \circ f\right|_{U_{j}}$.

From the assumption, $\left.g\right|_{V_{j}} \simeq_{\lambda, \eta} d_{j}$. Hence $\left.(g \circ f)\right|_{U_{j}}=\left.\left.\left.g\right|_{V_{j}} \circ f\right|_{U_{j}} \simeq_{\kappa, \eta} d_{j} \circ f\right|_{U_{j}} \simeq_{\kappa, \eta} \bar{d}_{j}$ where $\bar{d}_{j}:\left(U_{j}, \kappa\right) \rightarrow(Z, \eta)$ is some constant function. Hence cat ${ }_{\kappa, \eta}(g \circ f) \leq m$.

Proposition 3.8. If $f, g: X \rightarrow Y$ are $(\kappa, \lambda)$-homotopic, then cat $_{\kappa, \lambda}(f)=\operatorname{cat}_{\kappa, \lambda}(g)$.

Proof. It suffices to show that $\operatorname{cat}_{\kappa, \lambda}(f) \leq \operatorname{cat}_{\kappa, \lambda}(g)$. Let $\operatorname{cat}_{\kappa, \lambda}(g)=n$. Then there is a cover $\left\{U_{1}, \ldots, U_{n+1}\right\}$ of $X$ such that $\left.g\right|_{U_{j}}: U_{j} \rightarrow Y$ is $(\kappa, \lambda)$-nullhomotopic (that is, it is $(\kappa, \lambda)$-homotopic to a constant function $c_{j}:\left(U_{j}, \kappa\right) \rightarrow(Y, \lambda)$ for each $\left.j\right)$. Since $f \simeq_{\kappa, \lambda} g$, we have $\left.\left.f\right|_{U_{j}} \simeq_{\kappa, \lambda} g\right|_{U_{j}}$ for each $j$. Hence $\left.f\right|_{U_{j}}$ is $(\kappa, \lambda)$-nullhomotopic.

Corollary 3.9. Let $f: X \rightarrow Y$ be a $(\kappa, \lambda)$-continuous function. Then cat $_{\kappa, \lambda}(f)=0$ if and only if $f \simeq_{\kappa, \lambda} c$ where $c: X \rightarrow Y$ is a constant function.

Proof. This follows from Definition 3.1 and Proposition 3.8.

Theorem 3.10. If $f: X \rightarrow Y$ is $(\kappa, \lambda)$-homotopy equivalence, then $\operatorname{cat}_{\kappa, \lambda}(f)=\operatorname{cat}_{\kappa}(X)=$ $\operatorname{cat}_{\lambda}(Y)$.

Proof. By Remark 3.2, $\operatorname{cat}_{\kappa}(X)=\operatorname{cat}_{\kappa, \kappa}\left(\operatorname{Id}_{X}\right)$. Let $g$ be a homotopy inverse of $f$. That is, $g: Y \rightarrow X$ is a $(\lambda, \kappa)$-continuous function such that $g \circ f \simeq_{\kappa, \kappa} \operatorname{Id}_{X}$ and $f \circ g \simeq_{\lambda, \lambda} \operatorname{Id}_{Y}$. Then we have

$$
\operatorname{cat}_{\kappa}(X)=\operatorname{cat}_{\kappa, \kappa}\left(\operatorname{Id}_{X}\right)=\operatorname{cat}_{\kappa, \kappa}(g \circ f) \leq \operatorname{cat}_{\kappa, \lambda}(f) \leq \operatorname{cat}_{\kappa}(X) .
$$

Note that the second equality and the first and the second inequalities follow from Proposition 3.8, Proposition 3.7, and Theorem 3.6 respectively.

The assertion follows from Theorem 2.7.

Corollary 3.11. Let $f: X \rightarrow Y$ be a $(\kappa, \lambda)$-homotopy equivalence from a $\kappa$-contractible digital image (or to a $\lambda$-contractible digital image). Then cat $_{\kappa, \lambda}(f)=0$.

Proof. This follows from Theorem 3.10 .

Note that a set $X$ in $\mathbb{Z}^{n}$ is symmetric with respect to the origin if $X$ has the property that $x$ is an element in $X$ if and only if $-x$ is an element in $X$. If $X \subset \mathbb{Z}^{n}$ and $Y \subset \mathbb{Z}^{m}$ are two digital images and $X$ is symmetric, a map $f: X \rightarrow Y$ which satisfies $f(-x)=-f(x)$ for all $x \in X$ is called an antipodal map [9]. For the definition of a symmetric subset of an Euclidean space with respect to the origin and an antipodal map on it, see [12], pp. 261.

Example 3.12. Let $\mathrm{MSC}_{4}$ be a digital image in $\mathbb{Z}^{2} 4$-isomorphic to

$$
\left\{c_{0}=(1,-1), c_{1}=(1,0), c_{2}=(1,1), c_{3}=(0,1), c_{4}=(-1,1), c_{5}=(-1,0), c_{6}=(-1,-1), c_{7}=(0,-1)\right\}
$$

(see Figure 1). Then $\operatorname{cat}_{4}\left(\mathrm{MSC}_{4}\right)=1$. Note that this assertion appeared in [3], but the argument offered as proof in [3] only establishes that $\operatorname{cat}_{4}\left(\mathrm{MSC}_{4}\right) \in\{0,1\}$. To complete the proof, it suffices to show that cat $_{4}\left(\mathrm{MSC}_{4}\right) \neq 0$ and this follows from the observation that $\mathrm{MSC}_{4}$ is not 4-contractible [9]; Corollary 3.9, which shows that cat ${ }_{4,4}\left(\operatorname{Id}_{\mathrm{MSC}_{4}}\right) \neq 0$; and Remark 3.2.

Remark 3.13. The proof of $\operatorname{cat}_{4}\left(\mathrm{MSC}_{4}\right)=1$ also follows from Theorem 2.10 since $\mathrm{MSC}_{4}$ is a simple closed 4-curve. 


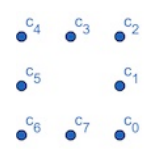

Figure 1. $\mathrm{MSC}_{4}$

The definition of wedge in digital topology was introduced in [16]. We refer to [8] for a corrected definition. Let $(X, \kappa)$ be a digital image such that $X=X_{0} \cup X_{1}$ where $X_{0} \cap X_{1}=\left\{x_{0}\right\}$ and if $x$ and $y$ are $\kappa$-adjacent for $x \in X_{0}$ and $y \in X_{1}$ then $x_{0} \in\{x, y\}$. In this case, $X$ is called the wedge of $X_{0}$ and $X_{1}$ and denoted $X=X_{0} \vee X_{1}$ and $x_{0}$ is called the wedge point.

Lemma 3.14. Let $X_{0}=X_{1}=\mathrm{MSC}_{4}$ and consider $X=X_{0} \vee X_{1}$ where $x_{0}$ is the wedge point of $X$. Then $X$ is not 4-contractible.

Proof. Assume that $X$ is 4-contractible. Then there exists a digital homotopy $H$ : $X \times[0, m]_{\mathbb{Z}} \rightarrow X$ such that $H(x, 0)=x$ and $H(x, 1)=x_{0}$. Consider the digital $(4,4)$ continuous function $r: X \rightarrow X_{0}$ where $r(x)=x$ for $x \in X_{0}$ and $r(x)=x_{0}$ otherwise. Then the digital map $G: X_{0} \times[0, m]_{\mathbb{Z}} \rightarrow X_{0}$ defined by $G(x, t)=r \circ H(x, t)$ gives a digital $(4,4)$-homotopy between an identity map on $X_{0}$ and a constant map at $x_{0}$ which is a contradiction with the fact that $\mathrm{MSC}_{4}$ is not 4-contractible [9].

Example 3.15. Consider the digital image $\mathrm{MSC}_{4} \vee \mathrm{MSC}_{4}$ with the wedge point $c_{0}$ as shown in Figure 2. It can easily be seen that the subsets $U=\left\{c_{4}, c_{3}, c_{2}, c_{1}, c_{0}, d_{1}, d_{2}, d_{3}, d_{4}\right\}$ and $V=\left\{c_{5}, c_{6}, c_{7}, c_{0}, d_{7}, d_{6}, d_{5}\right\}$ are 4-contractible and cover $\mathrm{MSC}_{4} \vee \mathrm{MSC}_{4}$ so that the inclusion maps $i_{U}: U \rightarrow \mathrm{MSC}_{4} \vee \mathrm{MSC}_{4}$ and $i_{V}: V \rightarrow \mathrm{MSC}_{4} \vee \mathrm{MSC}_{4}$ are both 4nullhomotopic. Hence cat $4\left(\mathrm{MSC}_{4} \vee \mathrm{MSC}_{4}\right) \leq 1$. By Lemma 3.14, the identity function on $\mathrm{MSC}_{4} \vee \mathrm{MSC}_{4}$ cannot have LS category value 0 for 4 -adjacency and it follows from Remark 3.2 that $\operatorname{cat}_{4}\left(\mathrm{MSC}_{4} \vee \mathrm{MSC}_{4}\right) \neq 0$. Hence $\operatorname{cat}_{4}\left(\mathrm{MSC}_{4} \vee \mathrm{MSC}_{4}\right)=1$.

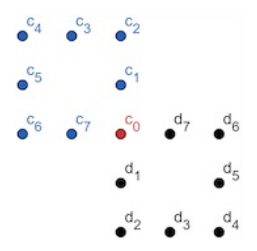

Figure 2. $\mathrm{MSC}_{4} \vee \mathrm{MSC}_{4}$

Example 3.16. Define the antipodal map $f:\left(\mathrm{MSC}_{4}, \kappa_{1}\right) \rightarrow\left(\mathrm{MSC}_{4}, \kappa_{2}\right), f\left(c_{i}\right)=c_{i+4(\bmod 8)}$ (see Figure 3). Note that $f$ is $\left(\kappa_{1}, \kappa_{2}\right)$-continuous whenever $\kappa_{2} \geq \kappa_{1}$ or $\kappa_{1}=\kappa_{2}$ where $\kappa_{1}, \kappa_{2} \in\{4,8\}$.

- Take $\kappa_{1}=\kappa_{2}=8$. Since $\mathrm{MSC}_{4}$ is 8 -contractible [15,16], $1 \simeq_{8,8} c$ where 1 and $c$ are the identity map and constant map respectively. Then $f \simeq_{8,8} f \circ c$, that is $f$ is also homotopic to a constant map. Hence cat $_{8,8}(f)=0$.

- Take $\kappa_{1}=4$ and $\kappa_{2}=8$. By Theorem 3.6 and Example 2.7 in [3], we have

$$
\operatorname{cat}_{4,8}(f) \leq \min \left\{\operatorname{cat}_{4}\left(\mathrm{MSC}_{4}\right)=1, \operatorname{cat}_{8}\left(\mathrm{MSC}_{4}\right)=0\right\}
$$

and hence $\operatorname{cat}_{4,8}(f)=0$.

- Take $\kappa_{1}=\kappa_{2}=4$. Since $f \circ f$ is the identity function, it follows from Theorem 3.10 that $\operatorname{cat}_{4,4}(f)=1$. 


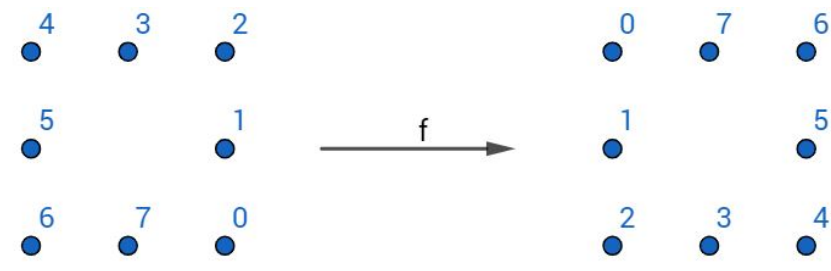

Figure 3. The antipodal map $f$ from $\mathrm{MSC}_{4}$ to itself.

\section{Digital LS category of digital diagonal map}

Definition 4.1. ([2,22]) For $(X, \kappa)$ and $(Y, \lambda)$, the normal (or strong) product adjacency, $N P(\kappa, \lambda)$, on $X \times Y$ is defined as follows. Two elements $\left(x_{1}, y_{1}\right)$ and $\left(x_{2}, y_{2}\right)$ in $X \times Y$ are $N P(\kappa, \lambda)$-adjacent if either

(i) $x_{1}=x_{2}$ and $y_{1}, y_{2}$ are $\lambda$-adjacent or

(ii) $y_{1}=y_{2}$ and $x_{1}, x_{2}$ are $\kappa$-adjacent or

(iii) $x_{1}, x_{2}$ are $\kappa$-adjacent and $y_{1}, y_{2}$ are $\lambda$-adjacent.

It is an easy exercise to see that the digital diagonal map $\Delta_{X}: X \rightarrow X \times X, \Delta_{X}(x)=$ $(x, x)$, is $(\kappa, N P(\kappa, \kappa))$-continuous.

Theorem 4.2. $\operatorname{cat}_{\kappa, N P(\kappa, \kappa)}\left(\Delta_{X}\right)=\operatorname{cat}_{\kappa}(X)$.

Proof. We can regard the function $\Delta_{X}$ as from $X$ to $\Delta_{X}(X)$. Let $\operatorname{pr}_{1}: \Delta_{X} \rightarrow X$ be the projection map $\operatorname{pr}_{1}(x, x)=x$. We see easily that $\operatorname{pr}_{1}$ is $(N P(\kappa, \kappa), \kappa)$-continuous and is the inverse of the function $\Delta_{X}$. Therefore, $\Delta_{X}$ is a $(\kappa, N P(\kappa, \kappa))$-isomorphism. The assertion follows from Theorem 3.10.

Acknowledgment. The authors would like to thank the anonymous referees for their helpful suggestions. In particular, we would like to thank the referee who contributed the improvement of the proof of $\operatorname{cat}_{4}\left(\mathrm{MSC}_{4}\right)=1$ in Example 3.12 and suggested computing the LS category of a simple closed $\kappa$-curve which contains more than four points.

\section{References}

[1] J.I. Berstein and T. Ganea, The category of a map and of a cohomology class, Fund. Math. 50, 265-279, 1961.

[2] C. Berge, Graphs and hypergraphs, 2nd edition, North-Holland, Amsterdam, 1976.

[3] A. Borat and T. Vergili, Digital Lusternik-Schnirelmann category, Turkish J. Math. 42 (4), 1845-1852, 2018.

[4] L. Boxer, Digitally continuous functions, Pattern Recognit. Lett. 15, 833-839, 1994.

[5] L. Boxer, A classical construction for the digital fundamental group, J. Math. Imaging Vision, 10, 51-62, 1999.

[6] L. Boxer, Properties of digital homotopy, J. Math. Imaging Vision, 22, 19-26, 2005.

[7] L. Boxer, Homotopy properties of sphere-like digital images, J. Math. Imaging Vision, 24, 167-175, 2006.

[8] L. Boxer, Digital Products, Wedges, and Covering Spaces, J. Math. Imaging Vision, 25, 159-171, 2006.

[9] L. Boxer, Continuous maps on digital simple closed curves, Appl. Math. 1, 377-386, 2010.

[10] L. Boxer, Alternate Product Adjacencies in Digital Topology, Appl. Gen. Topol. 19 (1), 21-53, 2018.

[11] O. Cornea, G. Lupton, J. Oprea and D. Tanre, Lusternik-Schnirelmann category, in: Mathematical Surveys and Monographs Vol. 103, American Mathematical Society, 2003. 
[12] T.T. Dieck, Algebraic Topology, European Mathematical Society, 2008.

[13] M. Farber, Topological complexity of motion planning, Discrete Comput. Geom. 29, 211-221, 2003.

[14] R.H. Fox, On the Lusternik-Schnirelmann category, Ann. of Math. 42, 333-370, 1941.

[15] S.E. Han, Computer topology and its applications, Honam Math. J. 25, 153-162, 2003.

[16] S.E. Han, Non-product property of the digital fundamental group, Inform. Sci. 171 (1-3), 73-91, 2005.

[17] G.T. Herman, Oriented surfaces in digital spaces, CVGIP: Graphical Models and Image Processing, 55, 381-396, 1993.

[18] I.M. James, On category in the sense of Lusternik-Schnirelmann, Topology, 17, 331$348,1978$.

[19] I. Karaca and M. Is, Digital topological complexity numbers, Turkish J. Math. 42 (6), 3173-3181, 2018.

[20] E. Khalimsky, Motion, deformation, and homotopy in finite spaces, Proceedings IEEE International Conference on Systems, Man, and Cybernetics, 227-234, 1987.

[21] L. Lusternik and L. Schnirelmann, Methodes topologiques dans les problemes variationnels, Institute for Mathematics and Mechanics, Moscow, 1930. (In Russian)

[22] G. Sabidussi, Graph multiplication, Math. Z. 72, 446-457, 1960.

[23] N.A. Scoville and W. Swei, On the Lusternik-Schnirelmann category of a simplicial map, Topology Appl. 216, 116-128, 2017.

[24] D. Stanley, On the Lusternik-Schnirelmann category of maps, Canad. J. Math. 54 (3), 608-633, 2002. 